\title{
REVUE DE LA LITTERATURE INTERNATIONALE
}

avec la collaboration de :

J. BELAISCH

M. BUVAT-HERBAUT

J.C. CZYBA

J.F. GUERIN

H. LEJEUNE

R.R. TREMBLAY 


\section{Revue de la littérature internationale}

\section{SPERMATOGENESE}

Physiopathologie de l'arrêt de la spermatogenèse R.C. MARTIN-DU-PAN, A. CAMPANA Clinique Stérilité, Hôpital Cantonal Universitaire de Genève, CH-1211, Genève, Suisse Fertil. Steril. Vol. 60 : 937-946, 1993.

\section{SPERMIOLOGIE}

Effet de l'héparine sur la migration du spermatozoïde humain in vitro L. SLIWA Department of Biology, Nicolaus Copernicus Academy of Medicine, 31-034 Krakow, Kopernika 7, Pologne Arch. of Andr., 30 : 177-181, 1993.

Une étude prospective des leucocytes et des sous-populations leucocytaires dans le sperme suggère qu'ils ne constituent pas une cause d'infertilité masculine M.J. TOMLINSON, C.L.R. BARRAIT, I.D. COOK

University of Sheffield, Department of Obstetrics and Gynaecology, Jessop Hospital for Women, Sheffield, and the infertility Unit, Royal Shrewsbury Hospital, Shrews bury, Shropshire, Royaume Uni

Fertil. Steril., Vol. 60 :1069-1075, 1993.

Mesure de paramètres endocriniens, biophysiques et biochimiques dans le plasma séminal obtenu par masturbation ou au cours d'une activité sexuelle N.V.SOKIKITIS, I.MIYAGAWA

Department of Urology, Tottori University School of Medicine, Yonago, Japan Journ. of Androl., Vol. 14 :366-373, 1993.

\section{ENDOCRINOLOGIE}

Exercice physique et fonction gonadique A.N. ELIAS, A.F. WILSON Department of Medicine, University of California at Irvine, CA 92717, USA. Hum. Repr., Vol. 8 : 1747-1761, 1993.

La mélatonine potentialise la suppression induite, par la testostérone de la sécrétion de LH chez des hommes normaux R.A. ANDERSON, G.A. LINCOLN, F.C.W. WU MRC Reproductive Biology Unit, Centre for Reproductive Biology, 37 Chalners Street, Edinburgh EH3 $9 E \mathrm{~W}$, Royaume Uni

Hum. Repr., Vol. 8 :1819-1822, 1993.

\section{THERAPEUTIQUE}

Le traitement de l'infertilité masculine est-il efficace ? revue et méta-analyses des essais randomisés publiés P.A. O'DONOVAN, P. VANDEKERCKHOVE, R.J. LILFORD, E. HUGUES

Institute of Epidemiology and Health Services Research, University of Leeds, Leeds, U.K et Mc Master University, Hamilton, Ontario, Canada

Hum. Repr., Vol. 8 : 1209-1222, 1993. 
Efficacité de la kallikréine comme traitement de l'oligozoospermie et de l'asthénozoospermie : un essai en double-aveugle M. GLEZERMAN, E. LUNENFELD, G. PotAShNick, M. Huleihel, Y. SofFer, S. SEGAL

Soroko Medical Center and Ben Gourion University Beer Sheba; Assaf Harofeh Hospital, Zrifin; Tel Aviv University and Barzilay Medical Center, Ashkelon. Israel

Fertil. Steril., Vol. 60 : 1052-1056, 1993.

L'acide folinique dans le traitement de l'infertilité masculine G. BENTIVOGLIO, F. MELICA, P. CRISTOFORONI

Istituto di Clinica Obstetrica e Ginecologica, Università di Genova, Genova, Italie Fertil. Steril., Vol. 60 : 698-701, 1993.

\section{FECONDATION IN VITRO}

Grossesse après fécondation avec des spermatozoïdes humains d'origine testiculaire R. Schoysman, P. Vanderzwalmen, M. NiJs, L. Segal, G. Segat-Bertin, L. GEERTS, E. VAN ROOSENDAAL, D. SchOYSMAN

Schoysman Infertility Management Foundation, IVF Laboratory, 1800 Vilvoorde, Belgique

The Lancet, Vol. 342 : 1327, 1993.

\section{SEXUALITE}

Effets de deux drogues hypotensives, l'aténolol (ténormine) et la mifedipine (adalate) sur la fonction sexuelle de l'homme âgé : une étude contrôlée avec cross-over D.L. MoRissetTe, M.H. SKINNER, B.B. HofFMAN, R.E. LEVINE, J.L. DAVIDSON Department of Molecular and Cellular Physiology, Stanford University, Stanford, California 943035-5426, USA

Arch. Sex. Beh., 22 : 99-108, 1993.

\section{CONTRACEPTION}

Comparaison de deux androgènes associés à la medroxy-progestérone pour l'obtention de l'azoospermie chez des Indonésiens

World Health Organization task force on methods for the regulation of male fertility Fertil. Steril., Vol. 60 : 1062-1068, 1993. 


\section{SPERMATOGENESE}

\section{Physiopathologie de l'arrêt de la spermatogénèse}

\author{
R.C. Martin-Du-Pan, A. CAMPana \\ Clinique Stérilité, Hôpital Cantonal \\ Universitaire de Genève, Suisse.
}

Fertil. Steril. Vol. 60, nº, 937-946, 1993.

L'arrêt de la spermatogenèse à différentes étapes du processus peut entraîner soit une oligospermie (arrêt partiel) soit une azoospermie (arrêt total).

Il s'agit d'une situation frustrante pour l'andrologue qui se trouve alors en présence d'un homme avec un volume testiculaire normal et chez lequel est suspectée une obstruction des voies spermatiques en raison du taux normal de FSH. Le diagnostic est fait par biopsie testiculaire. Divers auteurs ont constaté un arrêt de la spermatogenèse dans $4 \%$ à $30 \%$ des biopsies effectuées chez des patients présentant une oligospermie sévère ou une azoospermie.

Après avoir rappelé la physiologie de la spermatogenèse, les auteurs se livrent à une large revue de la littérature (89 références correspondant aux 35 dernières années).

\section{Différents niveaux de l'arrêt de la sper- matogenèse}

- Les spermatogonies. Le blocage de la multiplication des spermatogonies s'observe en cas d'insuffisance gonadotrope ou d'un traitement par un agoniste de GnRH. Cette situation est réversible.

Le même arrêt est obtenu après traitement par les antimitotiques ou la radiothérapie ; dans ce cas les valeurs de FSH sont très élevées.

- L'arrêt au stade spermatocytes $I$ est le plus souvent constaté à la fin de la prophase de lère division méiotique. Dans $70 \%$ des cas on peut relever des anomalies du déroulement de la prophase.

- L'arrêt au stade spermatide peut se produire à différentes étapes de la spermiogenèse.

\section{Les causes de l'arrêt spermatogénétique}

L'arrêt spermatogénétique peut être dû à des facteurs génétiques ou être la cause de l'action de différents facteurs.

\section{a) Les causes secondaires :}

- Causes iatrogènes : agonistes de GnRH, chimiothérapie, radiothérapie, antibiotiques (nitrofurantadine, gentamycine, niridazol).

- Facteurs nutritionnels et métaboliques : vitamine $A$ et déficiences en zinc, insuffisances hépatiques et rénales, anémie avec hématies falciformes.

- Chaleur : sauna, états fébriles ;

- Infections.

- Endocrinopathies : insuffisance gonadotrope, syndrome adrénogénital, hyperprolactinémie, syndrome de résistance aux androgènes, pseudohermaphrodisme mâle, anomalie de la chaine $B$ de LH.

- Causes testiculaires variées : varicocèle, torsion testiculaire, hydrocèle, cryptorchidie.

\section{b) Les causes primaires :}

- Aberrations du caryotype des cellules somatiques : XXY, XYY, trisomie, délétion de Yq11, translocation autosomique avec le chromosome $\mathrm{X}$ ou Y.

- Anomalies de la méiose aux stades zygotène, pachytène et diacinèse et à la métaphase $\mathbf{I}$.

\section{Essai thérapeutique avec GH-RH}

Jusqu'à présent aucun traitement ne s'est montré efficace dans l'azoospermie résultant d'un arrêt de la spermatogenèse, à l'exception de l'arrêt incomplet résultant d'une endocrinopathie ou de maladie infectieuse ou métabolique. Diverses études ayant montré le rôle joué par IGF1 sur la spermatogenèse, il a été envisagé de recourir à un traitement par l'hormone de croissance (GH) ou par GH-RH. GH stimule en effet la production hépatique de IGF1. Malgré les résultats encourageants obtenus dans une première étude par C. Moretti et $\mathrm{A}$. Fabbri, les auteurs dans une recherche personnelle portant sur 5 patients n'ont constaté aucun effet de GH$\mathrm{RH}$ sur la spermatogenèse.

\section{Commentaires (J.C. CZYBA)}

Il s'agit d'une excellente revue bibliographique des causes de bloquage de la spermatogenèse. Cette revue débouche sur le constat de l'absence actuel- 
le de tout traitement efficace, en raison semble-til d'une connaissance encore trop imparfaite des mécanismes locaux de la spermatogenèse. L'inventaire des facteurs autocrines et paracrines élaborés par la cellule de Sertoli et les cellules germinales est déjà impressionnant mais reste insuffisant.

Dans le même numéro de Fertility-Sterility ( $p$. 947-949) G.Huszar commente l'article de Martindu-Pan et Campana, en rappelant qu'à défaut de traitement permettant de restaurer la spermatogenèse existe maintenant la possiblité du recours à la fécondation assistée en utilisant soit des spermatozoïdes soit des spermatides. G.Huszar lance également un cri d'alarme et prône une large politique de prévention des troubles de la spermatogenèse induits par les nombreux toxiques industriels auxquels nous sommes exposés. Ces toxiques ne sont pas évoqués dans l'article que nous venons de résumer. Nous renvoyons le lecteur à l'article de M. Auroux et collaborateurs (page 13 de ce numéro d'Andrologie).

\section{SPERMIOLOGIE}

Effet de l'héparine sur la migration du
spermatozoïde humain in vitro

L. SLIWA

Department of Biology, Nicolaus Copernicus Academy of Medicine, Krakow, Kopernika 7, Pologne.

Arch. of Andr., 30 : 177-181, 1993.

Après l'ovulation le liquide folliculaire est transporté dans l'oviducte et chez divers mammifêres, il joue un rôle dans la capacitation, le déclenchement de la réaction acrosomique et dans la motilité des spermatozoïdes.

Plusieurs auteurs ont signalé l'existence d'un comportement chimiotactique du spermatozoïde humain induit par le liquide folliculaire in vivo et in vitro. L'héparine est présente dans le liquide folliculaire, la membrane cellulaire du spermatozoïde contient des récepteurs de l'héparine et l'héparine exerce in vivo et in vitro un effet sur la mobilité des spermatozoïdes.

Cette étude a pour propos de déterminer si l'héparine du liquide folliculaire exerce in vitro un effet chimioattractif sur le spermatozoïde humain.

\section{Matériel et Méthodes}

Seize échantillons de sperme ont été recueillis par masturbation, après 3 jours d'abstinence sexuelle, chez des donneurs en bonne santé, âgés de 22 à 40 ans. Pour un volume de 2 à $4 \mathrm{ml}$, les éjaculats contenaient au moins 40 millions de $\mathrm{sp} / \mathrm{ml}$ avec au moins $60 \%$ de formes normales et $60 \%$ de mobiles en trajets directs.

L'héparine a été dissoute dans du milieu BWW enrichi de $0,3 \%$ de sérum albumine.

Les essais ont été effectués à l'aide du dispositif suivant creusé dans des plaques de Peropex : 5 puits de $10 \mathrm{~mm}$ de diamètre et de $5 \mathrm{~mm}$ de profondeur, un au milieu et un dans chaque coin. Les puits périphériques sont reliés au puit central par une gouttière de $15 \mathrm{~mm}$ de long, $1 \mathrm{~mm}$ de large et $3 \mathrm{~mm}$ de profondeur. Le puit central a été rempli de $450 \mu \mathrm{l}$ de BWW et les puits péri- 
phériques du même volume de : BWW seul (contrôle), BWW + héparine à la concentration de $0,5 \mathrm{mg} / \mathrm{ml}$ (expérimental 1), de $0,05 \mathrm{mg} / \mathrm{ml}$ (exp.2) et de $0,005 \mathrm{mg} / \mathrm{ml}$ (exp. 3).

Après liquéfaction ( $30 \mathrm{~min}$ ) $0,2 \mathrm{ml}$ de sperme ont été introduits dans le puit central et les plaques ont été incubés à $36^{\circ} \mathrm{C}$ pendant 5 heures. Après incubation les spermatozoïdes ont été fixés dans le glutaraldehyde et comptés au microscope à contraste de phase.

\section{Résultats}

\begin{tabular}{|c|c|c|}
\hline Puits & $\begin{array}{l}\text { Concentration } \\
\text { de l'héparine } \\
(\mathrm{mg} / \mathrm{ml})\end{array}$ & $\begin{array}{l}\text { Nombre de spermatozoides } \\
\times 104 / 450 \mu l\end{array}$ \\
\hline central & 0 & $27,51 \pm 3,24$ \\
\hline contrôle & 0 & $4,92 \pm 1,37$ \\
\hline expérimental 1 & 0,5 & $5,94 \pm 2,03$ \\
\hline expérimental 2 & 0,05 & $10,45 \pm 2,15$ \\
\hline expérimental 3 & 0,005 & $8,44 \pm 1,76$ \\
\hline
\end{tabular}

L'augmentation de la concentration des spermatozoïdes dans les puits expérimentaux, par rapport au puit de contrôle, n'est significative que pour la concentration de $0,05 \mathrm{mg}$ d'héparine.

\section{Discussion}

Chez les espèces à fécondation externe l'œuf excrète des substances chimioattractives à des concentrations namomolaires. Le petit nombre d'informations sur l'existence et la nature de telles substances chez les mammiferes est dû à la difficulté d'étudier le comportement des spermatozoïdes in vivo et au manque de techniques convenables pour évaluer les effets de substances solubles in vitro.

Cette étude apporte la première démonstration, in vitro, de l'effet chimioattractif de l'héparine sur le spermatozoïde humain. Il semblerait que la fixation de l'héparine sur des récepteurs membranaires provoque des changements de la mobilité dont les modalités restent à étudier.

\section{Commentaires (J.C. CZYBA)}

Il s'agit d'une expérimentation élégante qui ne conclut pas cependant la recherche de substances attractives pour les spermatozoïdes humains. Nous avions nous-mêmes montré l'existence d'un comportement orienté des spermatozoïdes placés dans un gradient thermique (E. JEDDI, J.F. GUERIN, J.C. CZYBA - Have human spermatozoa a thermopreferendum? Biol. of Reprod., 16 ,
561-63, 1977). Il serait certainement intéressant de rechercher, grâce à l'analyse vidéo-micrographique assistée par ordinateur, la nature des modifications du mouvement des spermatozoïdes sous l'influence de substances comme l'héparine.

$\bullet \bullet \bullet$

\section{Une étude prospective des leucocytes et des sous-populations leucocytaires dans le sperme suggère qu'ils ne constituent pas une cause d'infertilité masculine}

\section{M.J. TOMLInson, C.L.R. BARRATT, I.D. COOK \\ University of Sheffield, Department of Obs- tetrics and Gynaecology, Jessop Hospital for Women, Sheffield, and the Infertility Unit, Royal Shrewsbury Hospital, Shrewsbury, Shropshire. Royaume Uni.}
Fertil. Steril., Vol.60, n 6 : 1069-1075, décembre 1993.

La relation entre la présence de leucocytes dans l'éjaculat et la fertilité masculine reste l'objet de controverse. De nombreuses études ont conclu à l'implication des leucocytes dans l'infertilité masculine, mais de façon indirecte, en montrant l'existence d'une corrélation entre la concentration des leucocytes et la dégradation des qualités du sperme. Deux études comparant la prévalence des leucocytes dans le sperme d'hommes fertiles et infertiles ont abouti à des conclusions opposées. Les travaux les moins récents n'utilisent pas les méthodes actuelles de discrimination entre leucocytes et "cellules rondes" c'est à dire cellules immatures de la lignée germinale.

Nous avons montré [1], contrairement à d'autres auteurs, que la présence de leucocytes dans l'éjaculat n'affecte pas les résultats de la FIV. Il nous a semblé logique de rechercher si la présence de cellules rondes (leucocytes et cellules germinales) est également en relation avec une diminution des chances de fécondation naturelle.

\section{Matériels et Méthodes}

Nous avons suivi pendant 2 ans l'évolution du spermogramme dans une population de 512 consultants pour infertilité. Nous avons égale- 
Tableau 1 : Semen parameters in 229 conceiving and nonconceiving couples in whom influence of pathology in the female was minimized.

\begin{tabular}{|c|c|c|}
\hline Semen parameter & $\begin{array}{l}\text { Conceiving } \\
(\mathrm{n}=54)\end{array}$ & $\begin{array}{c}\text { Nonconceiving } \\
(n=175)\end{array}$ \\
\hline $\begin{array}{l}\text { Motile density } \\
\left(\times 10^{6} / \mathrm{mL}\right)\end{array}$ & $22(3,68)$ & $4.5(0,58) \neq$ \\
\hline $\begin{array}{l}\text { Sperm density } \\
\left(\times 10^{6} / \mathrm{mL}\right)\end{array}$ & $55(13,117)$ & $23.5(5,117) \ddagger$ \\
\hline $\begin{array}{l}\text { Progressive } \\
\text { motility }(\%)\end{array}$ & $25(7,51)$ & $12(3,40) \div$ \\
\hline Ideal forms (\%) & $41(20,64)$ & $27(15,60) \ddagger$ \\
\hline $\begin{array}{l}\text { Total leukocytes } \\
\left(\times 10^{3} / \mathrm{mL}\right)\end{array}$ & $14(\mathrm{ND}, \S 262)$ & $13(1,244)$ \\
\hline $\begin{array}{l}\text { Neutrophils } \\
\left(\times 10^{3} / \mathrm{mL}\right)\end{array}$ & $7.5(\mathrm{ND}, 234)$ & $4.5(\mathrm{ND}, 149)$ \\
\hline $\begin{array}{l}\text { Macrophages } \\
\left(\times 10^{3} / \mathrm{mL}\right)\end{array}$ & $3(\mathrm{ND}, 48)$ & $4(\mathrm{ND}, 69)$ \\
\hline $\begin{array}{l}\text { HLA-DR-positive } \\
\text { cells }\left(\times 10^{3} / \mathrm{mL}\right)\end{array}$ & $2(\mathrm{ND}, 24)$ & $2(\mathrm{ND}, 20)$ \\
\hline $\begin{array}{l}\text { T4 lymphocytes } \\
\left(\times 10^{3} / \mathrm{mL}\right)\end{array}$ & $\mathrm{ND}(\mathrm{ND}, 3.8)$ & $\mathrm{ND}(\mathrm{ND}, 2.4)$ \\
\hline $\begin{array}{c}\text { T8 lymphocytes } \\
\left(\times 10^{3} / \mathrm{mL}\right)\end{array}$ & $\mathrm{ND}(\mathrm{ND}, 3.4)$ & $\mathrm{ND}(\mathrm{ND}, 3.0)$ \\
\hline $\begin{array}{l}\text { B lymphocytes } \\
\left(\times 10^{3} / \mathrm{mL}\right)\end{array}$ & $\mathrm{ND}(\mathrm{ND}, 4.0)$ & ND (ND. 2.0) \\
\hline $\begin{array}{l}\text { Interleukin-2-positive } \\
\text { cells }\left(\times 10^{3} / \mathrm{mL}\right)\end{array}$ & $\mathrm{ND}(\mathrm{ND}, 0.8)$ & $\mathrm{ND}(\mathrm{ND}, 2.0)$ \\
\hline $\begin{array}{l}\text { Reactive oxygen } \\
\text { species (mVs) }\end{array}$ & $18(1,719)$ & $19(1,406)$ \\
\hline $\begin{array}{c}\text { Immature germ celis } \\
\text { (cells } / 100 \text { sperm) }\end{array}$ & $11(3,30)$ & $14(4,69) \dagger$ \\
\hline
\end{tabular}

\footnotetext{
* Values are medians with $10 \%$ and $90 \%$ confidence limits in parenthesis.

+ Groups are significantly different, $P<0.05$.

$\ddagger$ Groups are significantly different, $P<0.0001$.

$\$ N D$, not detected.
}

ment quantifié dans le sperme : les diverses sous-populations de leucocytes, les cellules germinales immatures, les radicaux libres oxygénés et les anticorps antispermatozoïdes. Ont été exclus de l'étude les hommes dont les partenaires avaient une anomalie du bilan gynécologique, les azoospermiques et ceux qui présentaient une dysfonction sexuelle. Nous avons inclus 229 sujets, mais chez les 512 patients nous avons étudié la relation entre les souspopulations leucocytaires et les autres paramètres du sperme.

Les leucocytes ont été colorés par la méthode des phosphatases monoclonales alcaline et anti-alcaline décrite par Tomlinson et al [2]. Cette méthode nous permet de distinguer : neutrophiles, macrophages, cellules HLA-DR positives, lymphocytes $\mathrm{T} 4$, T8 et $\mathrm{B}$ et les interleukine-2 posi- tives. L'ensemble des leucocytes est révélé par l'anticorps HLe-1 et les cellules rondes non colorées sont considérées comme des cellules germinales immatures. Les radicaux libres oxygénés ont été mesurés par luminométrie (méthode de Kessopoulou et al.).

L'analyse statistique a été effectuée en ayant recours à la méthode de régression logistique (multivariée non paramétrique) et à la méthode de Cox.

\section{Résultats}

Dans les 229 couples retenus pour l'étude complète $54(23,5 \%)$ grossesses spontanées sont intervenues. Dans ce groupe les paramètres classiques du spermogramme et du spermocytogramme sont tous nettement plus élevés que dans le groupe resté infertile, tandis que le pourcentage de cellules immatures par rapport au nombre de spermatozoïdes est significativement plus bas. En revanche, il n'y a pas de différence significative entre les deux groupes en ce qui concerne la concentration des radicaux libres oxygénés ou d'une quelconque sous-population leucocytaire.

La régression logistique et l'analyse de Cox montrent que le pourcentage de spermatozoïdes mobiles, la mobilité progressive et la concentration des spermatozoïdes sont les paramètres les plus significativement prédictifs de la survenue d'une grossesse. Le pourcentage de formes normales et la concentration de neutrophiles ont également une valeur prédictive. Le taux des cellules immatures est associé négativement avec la survenue d'une grossesse. La concentration des radicaux libres et des anticorps (IgG ou IgA) n'a aucune relation avec la grossesse.

\section{a) Relations entre leucocytes et qualité du sperme}

Des leucocytes ont été mis en évidence chez $90 \%$ des 512 patients, essentiellement des neutrophiles et des macrophages. Il existe une corrélation positive entre la concentration de ces leucocytes et le taux de spermatozoïdes à forme normale et la numération ; cette corrélation correspond également à une association positive avec la grossesse. La concentration des radicaux libres oxygénés n'est correlée qu'avec le taux des neutrophiles et des macrophages.

\section{b) Leucocytospermie}

Nous avons relevé une leucocytospermie $>1 \times 10^{6}$ leucocytes/ml chez 14 des 512 patients dont 7 
individus du groupe de 229 - 3 de ces 7 individus ont été à l'origine d'une grossesse ( $43 \%$ contre $24 \%$ pour l'ensemble du groupe).

\section{c) Oligozoospermie}

Très peu de leucocytes ont été mis en évidence chez les hommes oligozoospermiques.

\section{d) Anticorps antispermatozoüdes}

Des anticorps ont été trouvés chez $11 \%$ des 512 patients. La présence d'anticorps n'est correlée ni avec les paramètres du sperme ni avec la survenue d'une grossesse.

Les cellules germinales immatures sont fortement corrélées avec de mauvais paramètres du spermogramme.

\section{Discussion}

Cette étude prospective est la première recherche systématique de l'influence des cellules rondes sur les paramètres du sperme et la survenue d'une grossesse.

En utilisant des méthodes d'analyse statistique particulièrement sensibles, nous avons montré que, contrairement à ce qui avait été établi par plusieurs auteurs, la présence de leucocytes n'affecte ni les paramètres du spermogramme ni la probabilité de survenue d'une grossesse. Il en va de même en ce qui concerne la concentration en radicaux oxygénés libres qui est, sans autre conséquence, seulement fortement correlée avec le taux de leucocytes.

En revanche nous avons montré que les cellules germinales immatures sont plus nombreuses dans le sperme des hommes qui restent infertiles. Leur présence est associée à de mauvais paramètres du spermogramme (oligo-asthéno et tératospermie) et traduit, selon nous, un mauvais déroulement de la spermatogenèse.

En opposition avec ce qui a été publié jusqu'ici nous avons ${ }_{3}$ montré que la présence de neutrophiles et de macrophages (leucocytes qui libèrent des radicaux libres oxygénées) améliore les paramètres du sperme et augmente la probabilité de grossesse. Ces leucocytes interviennent probablement pour éliminer les spermatozoïdes anormaux.

Nous estimons que la recherche du taux des leucocytes, préconisée de façon systématique dans l'examen de routine du sperme, est de peu d'intérêt. Nous suggérons de procéder à une réévaluation de la notion de stérilité masculine pour cause immunologique. Nous pensons enfin que l'évaluation de la présence et de la signification des cellules germinales immatures dans l'éjaculat mérite une attention particulière.

\section{Extrait de la bibliographie :}

1. Tomlinson MJ, BarRatT CLR, Bolton AE, Lenton EA, ALHASANI S., Diedrich K. Cooke ID. : Round cells and sperm fertilizing capacity : the presence of immature germ cells but not seminal leukocytes are associated with reduced success of in vitro fertilization. Fertil. Steril. $1992 ; 58: 1257-$ 1259 .

2. Tomlinson MJ, White A., Barratt CLR, Bolton AE, CoOke ID. : The removal of morphologically abnormal sperm forms by phagocytes : a positive role for seminal leucocytes? Hum. Reprod. $1992 ; 7: 517-522$.

\section{Commentaires (J.C. Czyba)}

Ce travail apporte des résultats surprenants dans la mesure où ils sont en contradiction avec la plupart (mais non toutes) des données de la littérature sur le sujet. L'ampleur de l'investigation et la rigueur de la méthodologie rendent difficile de prendre les auteurs en défaut. Il faut souligner ici l'intérêt de l'utilisation de nouvelles méthodes d'analyse statistique. Le recours de plus en plus fréquent à ces méthodes d'analyse multivariée apportera certainement encore bien des surprises.

\section{Commentaires (J.Belaisch)}

Cet article remet en question tant de notions que l'on croyait acquises, que l'on est heureux de voir qu'il confirme d'autres données solides, telles que des valeurs plus élevées des variables spermatiques : concentration, mobilité progressive et pourcentage des formes à morphologie idéale, dans le groupe des couples qui ont conçu par rapport aux autres!

\section{Mesure de paramètres endocriniens, biophysiques et biochimiques dans le plasma séminal obtenu par masturba- tion ou au cours d'une activité sexuelle}

\author{
N.V. SOFIKITIS, I. MIYAGAWA \\ Department of Urology, Tottori University \\ School of Medicine, Yonago, Japan. \\ Journal of Andrology, Vol. 14, 366-373, Sep- \\ tembre-Octobre 1993
}


Lorsque les techniques de reproduction assistée sont utilisées pour contrer une hypofécondité masculine, il importe d'obtenir le sperme de l'homme dans des conditions optimales. Ainsi se pose la question du mode de prélèvement de ce sperme : soit par masturbation dans un tube approprié, soit à la suite d'une rencontre sexuelle au cours de laquelle il y a éjaculation dans un condom fait de matière plastique.

\section{Matériel et méthodes}

Trente-huit (38) hommes dont l'âge a varié entre 23 et 37 ans ont participé à l'étude ; ils étaient considérés comme non féconds depuis au moins 3-5 années avec une partenaire présumée fertile.

Pendant une période de 20 à 25 jours, ces hommes ont déposé au laboratoire le spécimen de leur sperme obtenu par masturbation; par la suite, 6 autres échantillons ont été collectés dans un condom en silastique au cours d'une activité sexuelle.

Les paramètres habituels du spermogramme ont été évalués sur chacun des spécimens, au même titre que certains marqueurs des fonctions prostatiques, vésiculaires et épididymaires. Les résultats, présentés sous forme de tableaux, correspondent à la moyenne \pm 1 écart-type de 6 mesures individuelles d'un paramètre sélectionné pour les fins de l'étude.

\section{Résultats}

Le contenu du tableau 1 nous indique que la qualité globale des paramètres classiques du spermogramme est améliorée si l'éjaculat est obtenu au terme d'une rencontre sexuelle.
Par ailleurs, les parametres indicatifs de l'activité fonctionnelle des vésicules séminales (fructose et prostaglandine E), de l'épididyme ( $\alpha$-1,4-glucosidase) et de la prostate (citrate, zinc et cholestérol) sont présentés dans le tableau 2 ; seule l'activité prostatique paraît significativement augmentée dans l'éjaculat recueilli après une rencontre sexuelle.

Finalement, lorsque l'on fait subir un choc osmotique aux spermatozoides, lorsque l'activité de l'acrosine est mesurée et quand le nombre d'ovocytes de hamster pénétrés par les spermatozoides est exprimé en pourcentage, le sperme récolté dans un condom fait de silastique permet des appréciations nettement améliorées de ces 3 tests (tableau 3).

\section{Discussion}

Des évidences s'accumulent depuis 1985 en faveur de l'obtention du sperme dans un condom dont le matériel est dépourvu de toxicité pour les

\section{Tableau 3}

\begin{tabular}{|c|c|c|c|c|}
\hline & $n \S$ & $\begin{array}{l}\text { Swollen } \\
\text { sperm (\%) }\end{array}$ & $\begin{array}{c}\text { TAAS } \ddagger \\
\left(\mu \mid \mathrm{U} / 10^{6} \text { sperm }\right)\end{array}$ & $\begin{array}{l}\text { Penetrated } \\
\text { oocytes (\%) }\end{array}$ \\
\hline Masturbation & 38 & $38.4 \pm 5.6^{\circ}$ & $18.4 \pm 3.8^{*}$ & $4.1 \pm 1.7^{\circ}$ \\
\hline Intercourse & 38 & $54.3 \pm 8.1 \dagger$ & $27.3 \pm 4.1 \dagger$ & $14.4 \pm 3.2 \dagger$ \\
\hline
\end{tabular}

\section{Tableau I}

\begin{tabular}{lcccccccc}
\hline & & Total sperm & Volume & Motile & Motility & Normal & & \multicolumn{2}{c}{$\begin{array}{c}\text { Grade of sexual } \\
\text { satisfaction } \\
\text { (mf) }\end{array}$} & $\begin{array}{c}\text { sperm (\%) } \\
\text { content }\left(\times 10^{\circ}\right)\end{array}$ & $\begin{array}{c}\text { grade (0-4) } \\
\text { sperm (\%) }\end{array}$ & $\begin{array}{c}\text { pH } \\
(0-4)\end{array}$ \\
\hline Masturbation & 38 & $44.3 \pm 5.6 \dagger$ & $2.3 \pm 0.2 \dagger$ & $11.3 \pm 4.5 \dagger$ & $1.4 \pm 0.2 \dagger$ & $32.3 \pm 4.6 \dagger$ & $7.7 \pm 0.2 \dagger$ & $1.2 \pm 0.2 \dagger$ \\
Intercourse & 38 & $99.4 \pm 9.3 \ddagger$ & $3.3 \pm 0.2 \ddagger$ & $32.1 \pm 5.1 \ddagger$ & $2.7 \pm 0.2 \ddagger$ & $41.2 \pm 5.1 \ddagger$ & $7.1 \pm 0.2 \ddagger$ & $2.9 \pm 0.2 \ddagger$ \\
\hline
\end{tabular}

- Number of participants. For each participant, each parameter was assayed in all six samples obtained by each of the two semen collection methods (see Materials and Methods). Then the average of the six values for each parameter was calculated for each individual. The Mean and SD values shown in this table were calculated from the distribution of the $\mathbf{3 8}$ individual average values for each of the parameters.

$t$. $\neq$ Wittin each column, values not sharing the same superscript $(\dagger$ or $f)$ are significantly different $(P<0.001)$.

Tableau 2

\begin{tabular}{|c|c|c|c|c|c|c|c|}
\hline & \multirow[b]{2}{*}{$m$} & \multicolumn{3}{|c|}{ Prostate } & \multicolumn{2}{|c|}{ Seminal vesicte } & \multirow{2}{*}{$\begin{array}{l}\text { Epididymis } \\
\alpha \text {-glucosidase } \\
\text { (mU/ml) }\end{array}$} \\
\hline & & $\begin{array}{c}\text { Citrate } \\
n \ddagger(\mathrm{mg} / \mathrm{d})\end{array}$ & $\begin{array}{c}\text { Zinc } \\
\text { (mg/din) }\end{array}$ & $\begin{array}{c}\text { Cholesterol } \\
\text { (nmol/10' sperm) }\end{array}$ & $\begin{array}{l}\text { Fructose } \\
\text { (mg/dl) }\end{array}$ & $\begin{array}{c}\text { Prostaglandin E } \\
(\mu \mathrm{g} / \mathrm{ml})\end{array}$ & \\
\hline $\begin{array}{l}\text { Masturbation } \\
\text { Intercourse }\end{array}$ & $\begin{array}{l}38 \\
38\end{array}$ & $\begin{array}{l}296.4 \pm 40.2^{\circ} \\
387.3 \pm 44.2 \dagger\end{array}$ & $\begin{array}{l}14.2 \pm 1.2 \\
19.3 \pm 2.1 \dagger\end{array}$ & $\begin{array}{l}6.9 \pm 0.5^{\circ} \\
8.6 \pm 1.1 \dagger\end{array}$ & $\begin{array}{l}225.4 \pm 58.3^{\circ} \\
234.2 \pm 65.1^{\circ}\end{array}$ & $\begin{array}{l}88.5 \pm 9.6^{\circ} \\
91.2 \pm 8.3^{\circ}\end{array}$ & $\begin{array}{r}98.8 \pm 9.2^{*} \\
100.9 \pm 8.2^{*}\end{array}$ \\
\hline
\end{tabular}

if Within each colum, vatues not sharing the same superscript ( or $t)$ are significantly different $(P<0.001)$.

₹ Number of participants. Al of the parameters were assayed once in each of the subjects for each semen collection method. 
spermatozoides ; permettant une stimulation prolongée des organes sexuels parce gu'il y a eu coït, le sperme ainsi obtenu faciliterait la fertilisation des ovocytes in vitro et son évaluation globale permettrait une appréciation plus réaliste du pronostic de fécondité d'un homme qui tarde à démontrer sa paternite. Cet effet dit de stratégie de collecte de l'éjaculat sur la qualité du sperme et de ses divers indicateurs serait imputable à des stimulations hormonales, neurales et biochimiques du volume de la sécrétion prostatique. Dans un tel contexte, la masturbation pourrait être abandonnée au profit de l'éjaculation dans un condom pendant une rencontre sexuelle.

\section{Commentaire (Roland R. TREMBLAY, Canada)}

Les conclusions de cette étude sont supportées par un nombre adéquat d'observations et il s'en dégage une nette impression de cohérence. S'il y a un plaisir sexuel associé à l'éjaculation et par voie de conséquence si le nombre de contractions bulbocaverneuses est augmenté, meilleure sera la récolte de spermatozoides motiles pour fins de sélection dans un contexte de reproduction assistée. Il est intéressant d'imputer ce gain à une sécrétion prostatique accrue, et le $\mathrm{pH}$ mesuré en témoigne au même titre que le zinc, mais la mesure des kallicréines prostatiques (antigène prostatique spécifique, $\beta$-microseminoprotéine (PSP-94) et kallicréine glandulaire humaine (HGK-1) aurait apporté un éclairage précieux sur la fragmentation protéolytique des séménogélines qui se produit dans d'étroites fenêtres de $p H$. En effet, le jeu interactif de ces kallicréines pourrait-il être associé, de préférence au citrate et au cholestérol, à ce gain important (facteur de 3) de la mobilité totale des spermatozoides obtenus dans des conditions fort différentes au cours du travail de ces auteurs.

\section{ENDOCRINOLOGIE}

\section{Exercice physique et fonction gonadique}

\author{
A.N. EliAS, A.F. WILSON
}

Department of Medicine, University of California at Irvine, Irvine, USA.

Hum. Reprod., Vol. 8, 1747-1761, 1993.

\section{INTRODUCTION}

L'exercice physique s'accompagne d'une modification de la sécrétion d'un certain nombre d'hormones hypophysaires. Pour la plupart de ces hormones, on observe une augmentation sauf pour la $\mathrm{LH}$ qui diminue. La diminution de la sécrétion gonadotrope liée aux efforts physiques soutenus et répétés, est probablement la cause des anovulations observées chez les athlètes féminines sur-entraînées. Chez l'homme, la diminution de LH n'apparait pas réduire de manière aussi intense la concentration de testostérone.

La signification physiologique de la suppression de la sécrétion gonadotrope par l'exercice physique pourrait être que l'exercice stimule une réaction de défense visant à privilégier la survie de l'individu plutôt que la prolifération de l'espèce. Une dépression de la sécrétion de gonadotrophine chez la femme et l'infertilité qui en découle serait avantageuse pour la protection du groupe familial existant. Une modification similaire, mais moins intense, chez l'homme pourrait avoir le même but en réduisant sa libido ; la diminution moins profonde de la testostérone permettant toutefois de préserver la masse musculaire et de laisser intacte la possibilité de défense.

\section{Contrôle de la sécrétion des gonadotro- phines hypophysaires}

La régulation neuroendocrine de la sécrétion des hormones gonadotropes dépend de la sécrétion épisodique pulsatile de gonadotropin-releasing hormone (GnRH) par l'hypothalamus. La fonction de reproduction des mammiferes requière la survenue d'un épisode sécrétoire de GnRH environ toutes les $90 \mathrm{mn}$. Le ralentissement de la fréquence de la libération de $\mathrm{GnRH}$ provoque une diminution de la sécrétion de gonadotrophines responsable d'une aménorhée et d'une 
anovulation chez la femme et d'un hypogonadisme chez l'homme.

Chez l'homme, la sécrétion pulsatile de GnRH peut être mise en évidence chez le fotus à la 20 ème semaine de gestation, elle reste active chez le nouveau né puis disparaît chez l'enfant, jusqu'à la puberté. Une sensibilité accrue des neurones à $\mathrm{GnRH}$ au rétrocontrôle négatif exercé par les stéroïdes sexuels et une inhibition exercée par l'hormone pinéale, la mélatonine pourraient expliquer la suppression de la sécrétion de GnRH pendant l'enfance. La capacité de la glande pinéale à sécréter de la mélatonine apparaissant relativement constante, la concentration plasmatique de mélatonine diminue au cours de la croissance. On pense que lorsque la concentration de mélatonine est en dessous de 500 pmolll, la sécrétion pulsatile de $\mathrm{GnRH}$ est réactivée et la puberté est initiée. La réactivation de la sécrétion pulsatile du GnRH à la puberté est associée à une augmentation de la sécrétion des gonadotrophines qui survient de manière d'abord nocturne puis, avec l'avancée de la puberté, sur tout le nyctémère.

En plus du rétrocontrôle négatif des stéroïdes sexuels et de l'effet inhibiteur de la mélatonine, la sécrétion de GnRH est aussi influencée par de nombreux neurotransmetteurs et neuropeptides (Tableau 1).

La dopamine et le blocage adrénergique pharmacologique provoquent une diminution de la fréquence des pulses de GnRH. Les opioïdes endogènes et les narcotiques comme la morphine inhibent aussi la libération de $\mathrm{GnRH}$, cet effet peut être supprimé par les antagonistes des opiacés comme la naloxone. Une augmenta-

Tableau 1 : Facteurs neurohumoraux affectant la libération de $\mathrm{LH}$.

Facteurs stimulant la libération de LH

Acide gamma-aminobutyrique (GABA)

Vasoactive intestinal peptide (VIP)

Substance P

Facteurs inhibant la libération de LH

Dopamine

Blocage $\alpha$-adrénergique

Opioïdes : $\beta$-endorphine, [met]enkephaline

Corticotrophin releasing hormone (CRH)

Mélatonine

Prolactine (via dopamine)

Somatostatine

Cholecystokinine

Gastrine

Neurotensine tion du tonus dopaminérgique ou opioïde hypothalamique provoque une diminution de la sécrétion de GnRH. La Corticotrophin-releasing hormone ( $\mathrm{CRH})$ inhibe la libération de GnRH par un mécanisme que l'on pense être dépendant d'une augmentation du tonus opioïde central. Par de son effet de suppression de la libération de LH et de suppression du comportement sexuel, la CRH pourrait jouer un rôle important dans l'effet inhibiteur du stress sur la fertilité.

\section{Les syndromes d'hypogonadisme fonc- tionnel}

Chez la femme, les modifications de la fonction gonadique liées à l'exercice physique font partie d'un syndrome plus large d'aménorrhée hypothalamique fonctionnelle (i.e. sans anomalie anatomique de l'axe hypothalamo-hypophyso-ovarien). Ce syndrome est considéré comme non organique et réversible.

Le syndrome d'aménorrhée psychogène ou aménorrhée liée au stress est caractérisé par un déficit de sécrétion de GnRH comme démontré par le profil de sécrétion de $\mathrm{LH}$ avec diminution de l'amplitude et la fréquence des pulses. La phase de récupération est caractérisée par une réapparition du profil de sécrétion gonadotrope identique à ce qui est vu lors de la maturation pubertaire. L'administration de naloxone, antagoniste des récépteurs aux opioïdes ou de métoclopramide, antagoniste des récépteurs D2 dopaminérgiques, provoquent une augmentation de la concentration circulante de LH. Dans des expériences à long terme, la normalisation du profil sécrétoire de LH est souvent observée lors de la prise de métoclopramide ou l'utilisation de naltrexone, antagoniste de longue durée des opioïdes.

Les patientes présentant une aménorrhée psychogène présentent certaines caractéristiques des patientes dépressives, avec une hypersécrétion de cortisol pendant la journée et en début de soirée. La réponse de l'ACTH à la CRH est diminuée chez les patientes présentant une aménorrhée fonctionnelle, ce qui suggère une sécrétion endogène exagérée de $\mathrm{CRH}$. La dépression de la sécrétion de gonadotrophines dans l'aménorrhée fonctionnelle est probablement due à une sécrétion augmentée de CRH. L'effet inhibiteur de la $\mathrm{CRH}$ sur la sécrétion de GnRH peut être médiée par une augmentation du tonus opioïde central puisque antagonisé par la naloxone. 
Chez les hommes qui pratiquent un sport d'endurance (course) ou de résistance (haltérophilie), les concentrations sériques de testostérone, totale et libre, sont significativement plus basses que chez des sujets contrôles sédentaires. La diminution de la testostérone est néanmoins insuffisante pour avoir des conséquences cliniques aussi nettes que chez la femme. Des modifications des caractéristiques du sperme, en terme de concentration, mobilité et morphologie ne surviennent que chez les athlètes de sport d'endurance.

\section{Mécanisme}

L'exercice physique a depuis longtemps été reconnu comme une cause d'anomalies du cycle menstruel. Ces anomalies sont plus prononcées chez les femmes qui pratiquent des activités physiques plus soutenues comme la course de fond et la danse de ballet. Chez les athlètes pratiquant la course de fond, il existe une bonne corrélation entre le nombre de kilomètres parcourus et la sévérité des troubles menstruels. Toutefois, des exercices relativement modérés comme la pratique du jogging quand il est régulier, peut provoquer des irrégularités menstruelles avec anovulation ou insuffisance lutéale et diminution des pulses de $\mathrm{LH}$ en fréquence et en amplitude. L'intensité de l'exercice et le type d'activité physique ne sont pas les seuls facteurs qui peuvent intervenir dans les irrégularité menstruelles chez les athlètes. Le stress psychologique, la qualité de la nourriture comme la proportion respective d'hydrates de carbone et de protéines, le pourcentage de masse grasse par rapport au poids corporel peuvent venir perturber la relation entre l'intensité de l'exercice et les perturbations du cycle menstruel.

\section{a) Masse grasse et troubles menstruels}

La fréquence plus élevée des irrégularités menstruelles chez les coureuses de fond par rapport aux autres athlètes comme les nageuses ont été rapportées à la proportion de masse grasse significativement plus basse chez les coureuses de fond et les danseuses de ballet que chez les nageuses. Il a été proposé que la proportion de masse grasse requise pour une fonction menstruelle normale est d'environ $22 \%$ du poids corporel total. Chez les nageuses la proportion est proche de ce pourcentage critique, alors que chez les coureuses de fond et les danseuses la proportion de masse grasse est de $15 \%$ ou moins. Les réserves de graisse, particulièrement celles situés en dessous de hanches chez la femme, sont actuellement considérées comme capable d'influencer la sécrétion de GnRH par un mécanisme actuellement inconnu. Il a été postulé qu'une masse grasse suffisante peut influencer l'aptitude à la reproduction en représentant une source de conversion des androgènes en ostrogènes, en influençant le métabolisme des œestrogènes et en diminuant la sex hormone-binding globulin. Des réserves en graisse suffisantes indiquent que l'état nutritionnel est adéquat pour une grossesse et la lactation. Des réserves en graisse insuffisantes aboutissent par contre à une infertilité.

\section{b) Sécrétion des hormones hypophysaires après exercice physique}

L'exercice physique aigu est associé à une libération de plusieurs hormones hypophysaires. Certaines de ces hormones libérées au cours de l'exercice exercent un effet inhibiteur de la libération de $\mathrm{GnRH}$ hypothalamique (Tableau 2). La $\mathrm{LH}$, augmente de manière transitoire puis diminue. La FSH n'est habituellement pas modifiée par l'exercice particulièrement dans l'espèce humaine.

L'induction de la libération de GH et de prolactine par l'exercice peut être lié à une augmentation du tonus opioïde endogène puisque un traitement préalable par la naloxone diminue significativement l'augmentation de la GH et de la prolactine après l'exercice. Elle peut être en relation avec la production de lactate par l'anaérobiose induite par l'exercice, puisque reproduite par l'injection de L-lactate.

La prolactine a un effet inhibiteur de la sécrétion des gonadotrophines par une augmentation du tonus dopaminergique qui inhibe la sécrétion de GnRH. Cette augmentation du tonus dopaminergique vise à diminuer la concentration de prolactine mais a, de manière coïncidente, un effet d'inhibition de la libération de GnRH.

Tableau 2 : Relation entre la libération d'hormones par l'exercice physique et l'effet de ces hormones sur la sécrétion de GnRH/LH.

\begin{tabular}{|c|c|c|}
\hline Hortmone & $\begin{array}{l}\text { Modrication par l'exercice } \\
\text { physique }\end{array}$ & $\begin{array}{l}\text { Effet sur la sectétion de } \\
\text { GnRH et LH }\end{array}$ \\
\hline$\overline{G H}$ & 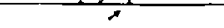 & $=$ \\
\hline Mélatonine & 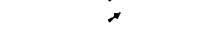 & $x$ \\
\hline $\mathrm{CRH}$ & 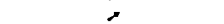 & 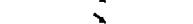 \\
\hline $\mathrm{ACTH}$ & 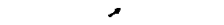 & $=$ \\
\hline B-lipoxophine & $\infty$ & $=$ \\
\hline B-endorphine & $71=$ & $x$ \\
\hline Prolactine & 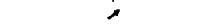 & $x$ \\
\hline Inhlbine & $=$ & $=$ \\
\hline
\end{tabular}




\section{c) Opioüdes endogènes et sécrétion des gona- dotrophines}

Il a été montré que la perfusion de $\beta$-endorphine diminue la sécrétion des gonadotrophines en agissant au niveau hypothalamique. L'augmentation de la sécrétion de LH par administration de naloxone suggère que les opioïdes exercent un effet tonique inhibiteur sur la sécrétion gonadotrope. Les dérivés de la proopiomélanocortine, $\beta$ endorphine et $\beta$-lipotrophine augmentent après un exercice aigu. Chez les femmes présentant une aménorrhée induite par l'exercice physique, le profil sécrétoire de LH est identique à celui des aménorrhées de stress pour lesquels les opioïdes semblent impliqués.

Dans des études réalisées chez des volontaires masculins, la concentration plasmatique de LH diminue de manière significative après un effort aigu. Chez les volontaires non entraînés, la diminution de LH s'accompagne d'une élévation de la $\beta$-lipotrophine mais pas de $\beta$-endorphine. Par contre, chez les hommes entraînés à l'exercice physique, une augmentation à la fois de la $\beta$ lipotrophine et de la $\beta$-endorphine a été constatée. Il est possible qu'une coupure plus rapide de la $\beta$-lipotrophine en $\beta$-endorphine chez les sportifs entraînés, soit avantageuse, ces sujets seraient ainsi mieux préparés pour lutter contre la douleur associée à l'exercice physique. Dans nos expériences, la diminution de la LH s'observe que les sujets soient entraînés ou non, en dépit d'une augmentation de la $\beta$-endorphine uniquement chez les sujets entraînés. Ceci nous amène à penser que la chute de LH induite par l'exercice n'est pas entièrement due à l'augmentation de la $\beta$-endorphine.

Certaines études, indiquent que l'intensité de l'effort physique et la libération de $\beta$-endorphine sont en relation. En plus de l'intensité de l'exercice, il apparaît que sa durée influence aussi la libération de $\beta$-endorphine qui se produit après 60 minutes d'exercice d'endurance.

Des stimuli générés lors du métabolisme anaérobique musculaire, augmentation de l'acide lactique, modification du $\mathrm{pH}$ sanguin, peuvent stimuler l'axe hypothalamo-hypophyso-surrénalien. Une diminution du $\mathrm{pH}$ dans les muscles par augmentation de la production de lactate provoque une augmentation réflexe de l'activité sympathique, et une libération de catécholamines, elles mêmes capables de stimuler la $\beta$ endorphine.
Chez les sujets entraînés, les niveaux d'insuline à l'état basal sont plus bas que chez les sujets non entraînés. Les concentration basses d'insuline à jeun sont connues pour augmenter le tonus opioïde, provoquant une activation du centre hypothalamique de la faim mais aussi une diminution de la pulsatilité du GnRH.

\section{d) $\mathrm{CRH}$ et sécrétion des gonadotrophines après exercice}

Comme la perfusion de CRH provoque une diminution de la concentration de $\mathrm{LH}$, nous avons étudié la relation entre l'élévation de la $\mathrm{CRH}$ induite par l'exercice et les gonadotrophines. Chez des hommes physiquement entraînés; la concentration de $\mathrm{CRH}$ augmente après un exercice aigu, avec un maximum juste après l'exercice. La concentration de LH diminue après l'exercice pour atteindre la valeur minimale entre 60 et 150 minutes.

Chez les athlètes féminines, la concentration moyenne de cortisol dans le sérum et l'excrétion urinaire de cortisol sont élevées. Ceci suggère que la sécrétion endogène de $\mathrm{CRH}$ est augmentée, ce qui peut inhiber la sécrétion de GnRH. Cet effet de la CRH peut être dépendant des opioïdes puisqu'il peut être aboli par les antagonistes des opiacés comme la naloxone.

\section{e) Mélatonine et sécrétion des gonadotro- phines après exercice}

Il est possible que les modifications de la concentration de mélatonine induites par l'exercice puisse jouer un rôle dans la diminution de la $\mathrm{LH}$. Physiologiquement, la lumière inhibe la sécrétion de mélatonine par un système mettant en jeu des neurones adrénergiques. Comme la glande pinéale est en dehors de la barrière hématoencéphalique, elle est exposée à l'augmentation des catécholamines circulantes lors de l'exercice physique. Un mécanisme par lequel la mélatonine peut modifier la fonction gonadique est lié à l'effet stimulant de la mélatonine sur la sécrétion de prolactine.

Si une élévation de la mélatonine est observée chez des sujets, hommes et femmes, après une course de longue distance, les valeurs atteintes sont toutefois inférieures à celles décrites comme inhibitrices de la sécrétion de GnRH ( $>500$ pmoUI). Par ailleurs, il n'y a pas, faute d'un temps suffisant pour une synthèse de novo, d'augmentation de mélatonine, après un exercice aigu qui provoque néanmoins une baisse de LH chez des hommes entraînés. 
Chez les athlètes féminines, les concentrations de mélatonine sont plus élevées pendant la journée que chez les femmes sédentaires. Chez les femmes ayant une aménorrhée induite par l'exercice, la concentration nocturne de mélatonine est significativement augmentée.

\section{FSH et inhibine après exercice}

L'exercice aigu ne diminue pas la concentration de FSH du sérum en dépit d'une diminution de la concentration de $\mathrm{LH}$ dans le même temps. Chez les hommes sportifs entraînés étudiés après effort aigu, nous n'avons pas pu démontrer, contrairement à des études chez l'animal, de diminution de la concentration de FSH ou d'inhibine par rapport à l'état basal avant exercice. Il est probable que, au moins chez l'homme, l'exercice physique ne provoque pas de libération d'inhibine.

\section{Conséquence de l'exercice sur la santé}

L'exercice physique régulier provoque des effets à la fois bénéfiques et délétères sur la santé. L'exercice physique régulier retarde l'augmentation des lipides avec l'âge, diminue la morbidité cardiovasculaire, préserve la masse osseuse, améliore la sensibilité à l'insuline et réduit le risque de cancer du sein. Les effets secondaires les plus significatifs de l'excès d'exercice physique sont l'ostéopénie et les anomalies des lipides.

\section{a) Ostéopenie}

L'exercice physique modéré entraîne une réduction de la perte osseuse après la ménopause. Toutefois, chez les athlètes en aménorrhée, le contenu minéral osseux est significativement abaissé par rapport à des femmes de même âge, non-aménorrhéique. La perte osseuse est probablement due à une réduction de la production d'œstrogènes. De plus, les voies du métabolisme des cestrogènes sont modifiées chez les athlètes en aménorrhée, avec augmentation des catécholœstrogènes inactifs.

\section{b) Effets sur les lipides}

De nombreuses études montrent que l'exercice physique régulier provoque une diminution des LDL et une augmentation des HDL dans le plasma, correspondant à une diminution du risque de maladies cardiovasculaires. Les athlètes en aménorrhée présentent toutefois une augmentation de l'apolipoprotéine $\mathrm{B}(\mathrm{LDL})$ relativement à l'apolipoprotéine A-I (HDL) et ces modifications tendent à annuler l'effet de cardioprotection de l'exercice chez ces sujets.

\section{c) Effets sur la croissance}

Il existe quelques démonstrations que l'effort physique intensif puisse provoquer un retard de croissance. L'entraînement intensif chez de jeune gymnastes féminines produit une diminution significative de l'insulin-like growth factor-I (IGF-I) et de la tri-iodothyronine (T3) après 3 jours d'entraînement. Ceci suggère que la diminution de l'IGF-I, l'effet anti-insuline de la libération de $\mathrm{GH}$ induite par l'exercice, la baisse de la T3 et l'élévation du cortisol peuvent ensemble contribuer à un retard de la croissance chez de telles athlètes.

\section{CONCLUSION}

Un exercice intense et aigu s'associe dans l'espèce humaine à une diminution significative de la concentration de $\mathrm{LH}$ mais à une absence de modification importante de la concentration de FSH. Les athlètes féminines entrainées, particulièrement celles qui ont des professions (danseuses de ballet) ou pratiquent des sports (course de marathon) demandant des efforts physiques prolongés, présentent des oligoménorrhées ou des aménorrhées. Les hommes ayant des activités physiques comparables ont des anomalies plus fines de l'axe hypophyso-gonadique avec des concentrations de testostérone inférieures à celles des sujets non entraînés. Le mécanisme de ces modifications hormonales reste imparfaitement compris. L'augmentation de l'acide lactique et les modification du $\mathrm{pH}$ lié à l'anaérobiose pendant l'effort pourraient être le mécanisme commun des modifications hormonales. Beaucoup des hormones libérées pendant l'exercice physique sont capables de déprimer la fonction gonadotrope. Pour plusieurs hormones, comme pour la mélatonine, l'augmentation n'est pas d'intensité suffisante pour expliquer complètement la dépression de la fonction gonadotrope. Néanmoins, la combinaison synergique des effets individuels des différentes hormones dont la libération est augmentée par l'exercice physique pourrait expliquer la diminution globale de la fonction gonadotrope.

\section{Commentaires (H. LEJEUNE).}

Cette revue générale fait le point à partir de 167 références bibliographiques sur les mécanismes des perturbations de la fonction de reproduction liées à la pratique du sport. On notera que les 
femmes apparaissent plus sensibles à l'effet néfaste de l'activité sportive sur l'axe gonadotrope, soit que l'altération de la fonction gonadotrope est plus facilement repérée chez elles par les troubles des règles, alors que chez l'homme la symptomatologie est absente, soit que les conséquences de l'effort physique soient plus marquée en raison de la plus grande complexité du fonctionnement gonadotrope cyclique féminin. L'association de l'effort physique à une baisse de la masse grasse n'est pas sans rappeler l'aménor-. rhée hypothalamique fonctionnelle qui accompagne la maigreur de l'anorexie mentale. On ne peut s'empêcher de rapprocher l'augmentation des opioüdes hypothalamiques avec la sorte d'addiction de certains pratiquant des sports d'endurance pour leur activité physique. On est finalement tenté de conclure que si une activité physique modérée est certainement bénéfique pour la santé, lorsque l'activité physique devient excessive et s'apparente $\grave{a}$ un trouble du comportement, elle devient néfaste, altérant en particulier la fonction gonadotrope, l'imprégnation en stéroïdes sexuels et la reproduction.

\section{$\bullet \bullet \bullet$}

\section{La mélatonine potentialise la sup- pression induite, par la testostéro- ne, de la secrétion de LH chez des hommes normaux}

\author{
R.A. Anderson, G.A. Lincoln, F.C.W. Wu \\ MRC Reproductive Biology Unit, Centre for \\ Reproductive Biology, 37 Chalners Street, Edin- \\ burgh EH3 $9 \mathrm{EW}$, Royaume Uni.
}

Human Repr., Vol. 8, 11 : 1819-1822, 1993.

Il a été montré que, dans l'espèce humaine, la sécrétion de mélatonine par l'épiphyse module l'activité de l'axe hypothalamo-hypophyso-gonadique. Chez la femme, l'administration de mélatonine, combinée ou non à un progestagène, diminue les taux sanguins de LH.

L'hypogonadisme hypogonadotrope chez un homme jeune a pu être mis en relation avec une sécrétion excessive de mélatonine. En revanche l'administration de mélatonine chez l'homme provoque une augmentation du taux de prolactine mais est sans influence sur les taux de LH, FSH et testostérone. Nous avons voulu étudier les effets de l'association testostérone-mélatonine.

\section{Matériels et méthodes}

Nous avons recruté 12 hommes en bonne santé âgés de 26 à 52 ans (moyenne 32 ans). Nous avons vérifié la normalité des taux plasmatiques de gonadotrophines et de testostérone.

Les sujets ont été répartis en deux groupes de six individus. Le premier groupe a été soumis à un traitement quotidien de $100 \mathrm{mg}$ de mélatonine pendant 14 jours et à une injection de $100 \mathrm{mg}$ de testostérone le 8ème jour ; deux mois après, les individus ont reçu pendant 14 jours un placebo et une injection de testostérone le 8ème jour. Le deuxième groupe a été soumis au traitement inverse.

Les concentrations de LH, FSH, testostérone, prolactine et mélatonine dans le plasma ont été déterminées, tous les deux jours par radioimmuno-assay.

Nous avons, d'autre part, vérifié que l'administration orale de $100 \mathrm{mg}$ de mélatonine augmente de façon spectaculaire sa concentration dans le sang.

\section{Résultats}

L'augmentation des taux de mélatonine dans le sang ne provoque pas de modification des taux de LH,FSH, prolactine et testostérone pendant les 8 premiers jours. L'association mélatoninetestostérone provoque dans la deuxième semaine une diminution des seuls taux de LH, significativement plus importante que chez les témoins (placebo+testostérone le 8ème jour).

\section{Discussion}

L'administration de doses quotidiennes supraphysiologiques de mélatonine ne modifie pas à elle seule, les sécrétions de l'appareil hypothalamo-hypophysaire. En revanche, ces doses modifient la sensibilité de l'appareil aux effets de la testostérone dont l'action freinatrice sur la sécrétion de $\mathrm{LH}$ est très importante au cours de la 2 ème semaine.

Cet effet est comparable à celui qui est obtenu chez la femme en associant mélatonine et progestérone. Le mécanisme d'action de la mélatonine reste discuté : effet direct sur la sensibilité des cellules neuro-sécrétoires ou effet sur le métabolisme de la testostérone, en particulier sur son aromatisation en estradiol? 
L'administration de mélatonine, à $16 \mathrm{~h}$ dans notre expérimentation, s'est accompagnée de sensations de fatigue chez certains sujets. Les conséquences n'ont pas été observées par les auteurs qui ont traité des femmes par de la mélatonine administrée à l'heure du coucher.

Nous suggérons d'étudier l'association de mélatonine à la testostérone dans un programme de contraception masculine, pour réduire les risques des doses élevées de testostérone.

\section{Commentaires (J.C. CZYBA)}

Les auteurs apportent une intéressante contribution à l'étude du rôle de la mélatonine dans la physiologie de la reproduction en constatant la symétrie de son action sur les appareils hypothalamo-hypophysaires masculin et féminin.

Cependant leur proposition finale d'utiliser la mélatonine dans un protocole de contraception hormonale masculine nous paraît hardie dans la mesure où elle vise à remplacer un risque (celui des fortes doses de testostérone) par un autre non encore évalué (perturbation de l'ensemble des rythmes circadiens sous l'influence d'un long traitement par de fortes doses de mélatonine).

\section{THERAPEUTIQUE}

\section{Le traitement de l'infertilité masculine est-il efficace? revue et méta-analyses des essais randomisés publiés}

\author{
P.A. O'Donovan, P. VANDEKERCKHOVE, R.J. \\ LILFORD, E. HUGHES
}

Institute of Epidemiology and Health Services Research, University of Leeds, Leeds, UK. \& McMaster University, Harnilton, Ontario, Canada.

Hum. Reprod., Vol. 8, 1209-1222, 1993.

Approximativement $10 \%$ des couples font l'expérience de l'infertilité et un facteur masculin est responsable de $30 \%$ des cas. La plupart des patients présentent une oligoasthénotératospermie de cause indéterminée pour laquelle les traitements sont variés et empiriques. Les essais thérapeutiques randomisés sont le seul moyen d'établir l'efficacité d'un traitement donné. Les principes en sont la présence d'un groupe contrôle, le tirage au sort et une étude statistique valide. Le but de ce travail est d'analyser les études publiées dans le domaine de l'infertilité masculine et de combiner les résultats de différentes études par la technique de la méta-analyse.

\section{Matériel et méthodes}

Les articles publiés dans le domaine de l'infertilité humaine entre 1966 et décembre 1990 ont été repérés par une recherche informatique sur Medline et une recherche manuelle dans 41 journaux. Les études portant sur l'infertilité masculine, comportant un groupe contrôle et analysant la survenue de grossesses ont été sélectionnées. La qualité des études a été analysée selon plusieurs critères : méthode de randomisation, taille de l'échantillon, groupes parallèles ou "crossover", description des sujets manquants, méthode de diagnostic des grossesses et expression du taux de grossesses par cycle ou par patient. Pour certains traitements, plusieurs études se sont révélées suffisamment comparables pour être analysées ensemble par méta-analyse. Trois types de méta-analyses ont été réalisées, $\mathrm{M} 1$ portant sur les seules études avec tirage au sort véritable et groupes parallèles, M2 incluant aussi les études avec pseudo-randomisation, M3 
Résultats des méta-analyses : 3 types de méta-analyses ont été réalisées : M1 : études avec tirage au sort, et groupes parallèles, M2: incluant de plus les études avec pseudo-randomisation, M3: incluant de plus les études en cross-over. Efficace : borne inférieure de l'intervalle de confiance à $95 \%$ de l'Odd Ratio supérieure à 1. ns: non significatif: borne inférieure de l'intervalle de confiance à $95 \%$ de l'Odd Ratio inférieure à 1 . Sont indiqués entre parenthèses (le nombre d'études; le nombre total de sujets inclus).
M1
M2
M3

Traitement de l'oligoasthénospermie

Anticestrogènes

Kallikrein

IAC-IU vs Coït

Bromocriptine

Androgènes ns

(4; 303)

Efficace

(3; 204)

ns

$(1 ; 98)$

ns

(3; 106)

ns

$(3 ; 585)$
Efficace

$(6 ; 504)$

Efficace

$(8 ; 556)$

Efficace

$(5 ; 344)$

Efficace

$(8 ; 1449)$ ns

$(4 ; 663)$

Efficace

$(4 ; 803)$
Sperme frais vs congelé

\section{IAD}

\begin{abstract}
LHu vs Temp
tenant compte en plus des études en cross-over. Pour chaque type de traitement, l'Odd-Ratio (OR) et son intervalle de confiance à $95 \%$ ont été calculés. L'OR exprime le "risque" relatif d'obtenir une grossesse dans le groupe traité par rapport au groupe contrôle. Si la borne inférieure de l'intervalle de confiance de l'OR est supérieure à 1 , le traitement est considéré efficace.
\end{abstract}

\section{Résultats}

Cent soixante quatorze études randomisées sur l'infertilité masculine ont été identifiées. Soixante douze examinant la survenue de grossesses ont été analysées individuellement, elles permettent rarement de conclure. En ce qui concerne le traitement de l'oligoasthénospermie, les résultats des méta-analyses montrent que les antiœstrogènes (tamoxifene ou clomifene), la Kallikrein donnée per os ou la réalisation d'IAC-IU se révèlent efficaces si l'on tient compte de l'ensemble des études analysables (M3). Si on ne tient compte que des études de meilleure qualité (M1), l'effet n'est plus significatif pour les antiostrogènes et les IAC-IU et est moins net pour la Kal- likrein. La bromocriptine, les androgènes (undécanoate de testostérone, mestérolone) n'apparaissent pas efficaces. En ce qui concerne les IAD, l'utilisation de sperme frais apparaît, sur l'ensemble des études (M3), plus efficace que le sperme congelé. La LH urinaire n'apparaît pas plus efficace que la courbe de température pour déterminer la date des IAD. Plusieurs questions importantes n'ont pas pu être examinées par méta-analyse comme l'intérêt du traitement des varicocèles, l'intérêt des corticoïdes dans les infertilités immunologiques. Aucune étude n'a comparé GIFT ou FIV à l'absence de traitement.

\section{Discussion}

Il y a peu d'études non biaisées dans le domaine de linfertilité masculine, surtout en ce qui concerne l'obtention de grossesses. Ceci contraste avec une vaste littérature sans groupe contrôle. La qualité de beaucoup des études randomisées est pauvre: peu d'études avec véritable tirage au sort, données souvent incomplètes, nombre de sujets faible, durée d'observation insuffisante pour juger de la survenue de grossesses, études croisées dans les- 
quelles les succès de la première phase ne sont pas soumis à la deuxième phase de traitement, ce qui favorise les résultats positifs. L'analyse des taux cumulatifs de grossesse par la méthode des courbes de survie et les études multicentriques avec randomisation centralisée sont exceptionnelles. Ainsi peu de conclusions peuvent être tirées. Les antiœstrogènes apparaissent potentiellement intéressant mais l'avantage n'est pas significatif si ne sont retenues que les études de bonne qualité. De plus larges études randomisées de bonnes qualités sont nécessaires pour conclure définitivement à un effet des antiœstrogènes. Nous sommes sceptiques sur l'efficacité de la Kallikrein qui parait efficace à la lecture superficielle de la méta-analyse. Toutefois, seules deux études sont véritablement randomisées et aucune multicentrique. Des études de meilleure qualité sont là aussi nécessaires. Les conclusions sur l'intérêt des IAC-IU sont entachées du fait que toutes les études sauf une sont en cross-over. La bromocriptine et les androgènes apparaissent inefficaces.

\section{Conclusion}

Le nombre et la qualité des études sur l'infertilité masculines sont insuffisants pour permettre de tirer des conclusions définitives dans la plupart des cas. Il y a un besoin pressant d'études véritablement randomisées, sur groupes parallèles, avec calcul de la puissance statistique, ayant une durée de suivi suffisante et si possible multicentriques.

\section{Commentaires (H. LEJEUNE)}

Alors que l'on pouvait espérer que la technique de la méta-analyse puisse enfin permettre des conclusions claires en cumulant les résultats de plusieurs études, ce travail souligne une fois de plus que l'efficacité ou l'inefficacité des traitements est très difficile à mettre en évidence dans le domaine de l'infertilité masculine. Selon les auteurs, c'est la mauvaise qualité des études qui est responsable de ces difficultés. On notera que le cahier des charges à remplir pour qu'une étude satisfasse au "gold standard" des auteurs est particulièrement lourd et on peut se demander si de telles études sont réalisables en pratique. Ce travail permet tout de même aux auteurs de conclure que, parmi les traitements médicamenteux de "l'oligoasthénospermie idiopathique", les antiostrogènes paraissent les plus susceptibles d'être efficaces. Malgré de bons résultats dans les métaanalyses, les auteurs apparaissent plus sceptiques quant à l'efffcacité de la Kallikrein.
Deux aspects pouvant rendre compte des difficultés à conclure quant à l'efficacité ou l'inefficacité d'un traitement méritent un commentaire :

$1^{\circ}$ Il existe une hétérogénéité des situations englobées dans le terme "oligoasthénospermie idiopathique" du fait de mécanismes physiopathologiques différents. On peut ainsi se demander s'il faut s'acharner à vouloir traiter différentes pathologies par le même médicament. On connaît des cas où un médicament donné se révèle efficace chez un patient donné. Par exemple, le patient décrit par Gooren (1989) a vu son spermogramme s'améliorer et une grossesse survenir à chaque fois qu'il était traité par le tamoxifene, l'oligoasthénospemie se renouvelant lors de l'arrêt du traitement. Ce patient présentait une insensibilité partielle aux androgènes. Un effort de classification des patients selon des critères physiopathologiques plus précis que "loligoasthénospermie idiopathique" est certainement un aspect à ne pas négliger dans les études sur l'infertilité masculine. Des efforts de recherche concernant les mécanismes physiopathologiques en cause dans les oligoasthénospermies dites "idiopathiques" pourraient constituer une solution vers la mise en évidence de l'effet de certains traitements dans certaines situations mieux définies.

$2^{\circ}$ L'attitude consistant à considérer comme informatives uniquement les études ayant pour critère de jugement le nombre de grossesses semble poser problème. On acquière de plus en plus clairement la notion que ce critère de jugement est entaché d'une telle variabilité "résiduelle" (due à la multiplicité des facteurs masculins et féminins intervenant dans l'obtention d'une grossesse) que l'effet du traitement masculin est noyé dans la variabilité due aux autres facteurs. Le travail présenté ici semble nous dire que les conditions qui permettraient de s'affranchir de la variabilité résiduelle $d u$ critère de jugement "grossesse" (nombre de cas, durée d'observation...) sont telles que les études sont pratiquement impossibles à réaliser. Il n'est pas impossible que les essais thérapeutiques s'adressant à des marqueurs moins variables permettent des conclusions que ne permettent pas d'obtenir les études jugeant de l'obtention de grossesses. Peut-être vaut-il mieux savoir que dans telle situation bien définie sur le plan physiopathologique, tel traitement améliore tel marqueur corrélé à la "fertilité masculine" et rester dans l'ignorance actuelle de l'effet des traitements de l'oligoasthénospermie idiopathique sur le nombre de grossesses.

\section{REFERENCE}

1. GOOREN L. Improvement of spermatogenesis after treatment with the antiestrogen tamoxifen in a man with the incomplete androgen insensibiity syndrome. J Clin Endocrinol Metab. 1989; 68 : 1207-1210. 
Efficacité de la Kallikreine comme traitement de l'oligozoospermie et de l'asthénozoospermie : un essai en double-aveugle

M. Glezerman, E. LunENFELd,G. POTASHNIK,BM. HULEIHEL, Y. SOFFER, S. SEGAL

Soroko Medical Center and Ben Gurion University, Beer Sheba; Assaf Harofeh Hospital, Zrifin ; Tel Aviv University and Barzi-

lay Medical Center, Ashkelon. Israel.

Fertil. Steril., Vol. 60, ${ }^{\circ} 6,1052-1056$, décembre 1993.

Ishigami et al. ont postulé que la kallikréine et ses effecteurs, kalidine et bradykinine, augmentent le nombre et la mobilité des spermatozoïdes et sont également responsables de leur intégrité morphologique. Pendant les 15 dernières années de nombreuses études cliniques ont montré que le traitement par la kallikréine améliore les paramètres des spermes déficients, en particulier la numération et la mobilité.

Le but du présent travail était de confirmer et préciser les effets d'un traitement par la kallidinogénase (qui libère de la kallikréine), administrée par voie orale à des hommes infertiles, sur les paramètres du sperme.

\section{Matériels et Méthodes}

Nous avons étudié 200 patients présentant une infertilité primaire ou secondaire supérieure à 1 an et âgés de 18 à 45 ans, sans traitement depuis plus de 2 mois, avec une numération inférieure à $20 \times 106 / \mathrm{ml}$ et/ou une mobilité inférieure à $30 \%$.

Le traitement a été administré pendant 12 semaines sous la forme de 2 comprimés 3 fois par jour. Pour la moitié des patients chaque comprimé contenait 100 unités de kallikréine, pour l'autre moitié un placebo d'aspect et de goût identiques.

Les spermogrammes ont été effectués toutes les six semaines à partir du début du traitement.

\section{Résultats}

Nous n'avons observé aucune amélioration de la numération et de la mobilité sous l'effet du traitement. Seule a pu être notée une amélioration significative de la mobilité dans le groupe placebo ! Le traitement a été bien supporté et sans effet sur les paramètres sanguins.

\section{Discussion}

Sur des bases empiriques la kallikréine est largement utilisée dans de nombreux pays comme traitement de l'infertilité masculine, avec des fortunes diverses mais un résultat considéré, dans l'ensemble, comme positif. Nos résultats sont clairement négatifs. Nous admettons que le système kallikréine-kinine joue probablement un rôle important dans la fonction reproductive mâle et, au moins théoriquement, dans la motilité des spermatozoïdes. Nous émettons l'hypothèse que des doses de kallikréine beaucoup plus importantes que celles qui sont couramment utilisees pourraient avoir un effet thérapeutique.

\section{Commentaires (J.C CZYBA)}

L'une des critiques adressées à la métaanalyse est que les résultats négatifs sont beaucoup plus rarement publiés que les résultats positifs alors que leur valeur est la même.

Ce travail remet en cause non seulement un certain nombre de publications tout à fait sérieuses mais également une pratique thérapeutique largement répandue. Les auteurs, avec une élégante modestie, se contentent de suggérer une augmentation des doses et se gardent d'amorcer la moindre étude critique de la littérature. Leur parti pris de prudence les conduits même à choisir un titre qui annonce le contraire de leur conclusion.

\section{L'acide folinique dans le traitement de l'infertilité masculine}

\author{
G. Bentivoglio, F. Melica, P. CRistoforoni \\ Istituto di Clinica Obstetrica e Ginecologica, Uni- \\ versità di Genova, Genova, Italie.
}

Fertil. and Steril., Vol. 60, 4 : 698-701, 1993.

L'acide folique (acide pteroylglutamique) et les folates participent à la synthèse de l'acide thymidylique (essentiel dans la régulation de la synthèse du DNA) à la synthèse des bases puriques et au métabolisme des acides aminés; de plus ils interfêrent dans l'action de la vitamine B12. 
L'acide folinique (métabolite de l'acide folique) est couramment employé dans le traitement des déficiences en folates et comme protecteur dans les thérapies par les antimétabolites et la radiothérapie. Son utilisation réduit la toxicité médullaire et protège les épithéliums de la desquamation. Des études récentes ont montré un effet favorisant de l'acide folique sur la multiplication et la maturation cellulaire.

Chez l'homme infertile, on observe fréquemment la présence dans l'éjaculat de quantités plus ou moins importantes de cellules germinales desquamées (spermatides et spermatocytes). La présence de nombreuses cellules rondes n'est pas toujours en relation avec une atteinte testiculaire (varicocèle, hydrocèle, inflammation) ou une affection systémique (infectieuse, hépatique, rénale, hormonale) et on est parfois en présence d'un "syndrome idiopathique des cellules rondes".

\section{Matériels et Méthodes}

Ont été étudiés 65 hommes infertiles et présentant un syndrome idiopathique des cellules rondes. Leurs conjointes avaient un bilan gynécologique normal. Ces hommes ont été traités par l'acide folinique seul, à la dose de $15 \mathrm{mg}$ une fois par jour, pendant 3 mois. Spermogramme et spermocytogramme ont été effectués au moins deux fois avant le début et après la fin du traitement.

Par ailleurs nous avons entrepris une expérimentation animale dans le but de mieux comprendre les effets de l'acide folinique sur l'histologie du testicule. Il n'y a pas de modèle animal correspondant au syndrome des cellules rondes, mais nos observations sur le testicule et l'éjaculat d'hommes recevant des drogues cytotoxiques nous avaient montré des similitudes avec ce syndrome. Nous avons traité pendant 14 jours des rats Wistar adultes par du Méthotrexate (MTX) ou, pendant 5 à 6 jours, par du Cyclophosphamide (CTX). Après l'arrêt du traitement, les rats survivants ont été pour moitié traités par l'acide folinique et pour moitié non traités. 14 jours après l'arrêt du traitement initial, les rats ont été sacrifiés et leurs testicules prélevés pour examen histologique.

\section{Résultats}

Dans les 6 mois suivant l'arrêt du traitement des 65 hommes infertiles, 24 grossesses ont été diagnostiquées chez leurs conjointes. Tous les paramètres du sperme ont été améliorés par le traitement (Tableau 1).
Tableau 1 :

\begin{tabular}{lcc}
\hline Evolution du sperme & $\begin{array}{c}\text { Avant le } \\
\text { traitement }\end{array}$ & $\begin{array}{c}\text { Après le } \\
\text { traitement }\end{array}$ \\
\hline $\begin{array}{l}\text { Nombre moyen de } \\
\text { spermatozoïdes en } \\
\text { millions/ml }\end{array}$ & $15,02 \pm 1,95$ & $22,58 \pm 2,61$ \\
$\begin{array}{l}\text { Nombre moyen de } \\
\text { cellules rondes } \\
\text { en millions/ml }\end{array}$ & $9,67 \pm 0,65$ & $6,36 \pm 0,49$ \\
$\begin{array}{l}\text { Rapport } \\
\text { Spermat/cell.rondes }\end{array}$ & 1,63 & 3,51 \\
Mobilité & $17,7 \%$ & $27,8 \%$ \\
\hline
\end{tabular}

Tableau 2 :

\begin{tabular}{lcc}
\hline $\begin{array}{l}\text { Amélioration des } \\
\text { paramètres du } \\
\text { sperme entre le début } \\
\text { et la fin du traitement }\end{array}$ & $\begin{array}{c}\text { Hom. devenus } \\
\text { fertiles (24) }\end{array}$ & $\begin{array}{c}\text { Iom. restés } \\
\text { infertiles (41) }\end{array}$ \\
\hline $\begin{array}{l}\text { Nombre de } \\
\text { spermatozoïdes }\end{array}$ & $99 \%$ & $65 \%$ \\
$\begin{array}{l}\text { Nombre de } \\
\text { cellules rondes }\end{array}$ & $49 \%$ & $29 \%$ \\
$\begin{array}{l}\text { Rapport Spermatoz. } \\
\text { sur cell.rondes }\end{array}$ & $248 \%$ & $78 \%$ \\
Mobilité & $128 \%$ & $32 \%$ \\
\hline
\end{tabular}

Les résultats sont encore plus spectaculaires si l'on compare les conjoints des femmes devenues enceintes et les hommes des couples restés infertiles (Tableau 2).

Chez les animaux, le traitement par l'acide folinique a amélioré l'activité mitotique de l'épithélium germinal et la cohésion cellulaire.

\section{Discussion}

Le rôle indiscutable de l'acide folinique dans les processus biologiques qui requièrent un index mitotique élevé, ainsi que l'absence d'effets secondaires, nous a poussé à rechercher son action chez les patients présentant un syndrome idiopathique des cellules rondes. Nous avons observé une amélioration significative des paramètres du sperme étudiés. Nos observations histologiques après expérimentation animale nous font supposer que l'acide folinique agit en freinant la desquamation des cellules immatures de la lignée germinale. 
Commentaires (J.C. CZYBA)

L'hypothèse de travail repose sur le constat, effectué par les auteurs, de caractéristiques identiques du spermogramme et de la biopsie testiculaire chez les hommes traités par des drogues cytotoxiques et ceux qui présentent un syndrome idiopathique des cellules rondes. A travers un raisonnement du type "aux mêmes effets les mêmes causes", ils estiment que ce qui est bon pour l'un devrait l'être pour l'autre. Le spermiologue plein d'expérience reste perplexe devant la simplicité de cette approche et continue de penser que l'étiquette idiopathique ne sert qu'à désigner le fourretout des anomalies restées sans explication.

On remarquera, pour la déplorer, l'absence de groupe témoin, sans s'étonner cependant de cette aberration qui est de règle dans la plupart des publications scientifiques sur les traitements de linfertilité.

L'acide folinique est préféré à l'acide folique dans la prévention des accidents toxiques provoqués par le méthotrexate et autres agents antifoliques qui agissent en bloquant la dihydrofolate-réductase des tissus. Dans la mesure où le syndrome idiopathique des cellules rondes n'est évidemment pas la conséquence d'un traitement par les agents antifoliques, on peut se demander si les auteurs n'auraient pas obtenu les mêmes résultats en ayant recours directement à l'acide folique. Il ne serait pas sans intérêt de rechercher chez les patients une éventuelle carence en acide folique.

Puisque, tout à fait généralement, la présence de cellules germinales immatures dans le sperme est la conséquence de leur desquamation, pourquoi ne pas rechercher systématiquement l'éventuel effet bénéfique d'un traitement par l'acide folinique ou folique chaque fois que l'on constate un taux élevé de ces cellules dans l'éjaculat?

Quoi qu'il en soit, les traitements efficaces de l'infertilité masculine sont rares et reposent le plus souvent sur des bases scientifiques mal établies. Les nouvelles propositions sont toujours bienvenues, surtout lorsqu'il s'agit d'un traitement économique (moins de 200 francs pour 3 mois) et sans effets secondaires. Il serait intéressant que d'autres équipes entreprennent de vérifier les résultats obtenus par G. Bentivoglio et ses collaborateurs.

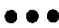

\section{FECONDATION IN VITRO}

\section{Grossesse après fécondation avec des spermatozoïdes humains d'origine testiculaire}

R. Schoysman, P.VAnderzWalmen, M.NiJs, L.Segal, G. Segal-Bertin, L.GEerts, E. VAN ROOSENDAAL, D.SCHOYSMAN

Schoysman Infertility Management Foundation, IVF Laboratory, Vilvoorde, Belgique

The Lancet, Vol 342 : 1237, Nov 1993.

L'aspiration microchirurgicale de spermatozoïdes épididymaires (AMSE) se solde parfois par une ponction blanche. Dans la mesure où des succès ont été rapportés chez l'animal, les auteurs proposent d'utiliser pour fécondation assistée des spermatozoïdes extraits de biopsies testiculaires.

\section{Matériel et Méthodes}

L'étude a porté sur 6 patients, présentant une agénésie déférentielle pour 5 d'entre eux, chez qui une AMSE a été pratiquée en même temps qu'une biopsie testiculaire. L'AMSE a été positive pour les 3 premiers (1-3) et négative pour les 3 derniers (4-6). Les tubes séminiferes ont été écrasés entre 2 lames de verre dans du Earle supplémenté en sérum, et les spermatozoïdes récupérés ont été centrifugés sur mini gradient de Percoll. Deux techniques de fécondation assistée ont été pratiquées : injection sous zonale (SUZI) ou intracytoplasmique (ICSI) de spermatozoïdes (spz). Dans SUZI, les spermatozoïdes ont été incubés avec de la pentoxifylline et du fluide folliculaire, et un nombre maximal de 10 ont été injectés dans l'espace périvitellin.

\section{Résultats}

Patients 1 à 3 : fécondation avec spermatozoïdes testiculaires : $5 / 11$ ovocytes.

Patients 1 à 3 : fécondation avec spermatozoïdees épididymaires : $9 / 11$ ovocytes.

Patients 4 à 6 : fécondation avec spermatozoïdes testiculaires : $4 / 24$ ovocytes.

Une grossesse évolutive a été obtenue à partir de 2 embryons. 


\section{Discussion}

Il s'agit de la 1 ère grossesse rapportée chez l'homme consécutive à une fécondation par des spermatozoïdes d'origine testiculaire; ce succès repose le problème de la maturation des spermatozoïdes dans les voies génitales mâles, qui parait facultative en technique de fécondation assistée.

\section{Commentaires (J.F. GUERIN)}

Cet article prouve indiscutablement que des spermatozoides d'origine testiculaire peuvent être fécondants, dans les situations d'agénésie déférentielle. (ll n'est absolument pas permis d'extrapoler aux situations physiologiques). La preuve de la grossesse n'est pas absolue, car à notre connaissance - et on peut regretter que cela n'apparaisse pas clairement dans le texte - les 2 embryons à l'origine de la grossesse évolutive étaient d'origine mixte. Il en est de même pour le cas rapporté par les mêmes auteurs dans une "lettre à l'éditeur" parue dans "Human Reproduction" (Vol 8, 1339-1340, 1993) : une grossesse unique après transfert de 3 aufs fécondés, $2 \grave{a}$ partir de spermatozoides épididymaires et 1 à partir de spermatozoides testiculaires. Mais à n'en pas douter, des enfants naîtront bientôt à partir de spermatozoides prélevés uniquement dans le testicule.

Le fait encore plus surprenant est que ces fécondations peuvent être obtenues par différentes techniques: ICSI, SUZI, et même FIV classique comme cela a été rapporté par Hirst et coll dans le même numéro du Lancet (1993). Or les techniques de FIV et même, dans une moindre mesure, de SUZI, nécessitent une certaine maturité membranaire de la part des spermatozoides au moins pour fusionner avec la membrane ovocytaire. Il nous faut admettre que, dans ces situations d'agénésie, une certaine maturité des spermatozoides est acquise dès leur libération dans la lumière des tubes séminiferes.

\section{SEXUALITE}

Effets de deux drogues hypotensives, l'Atenolol (Ténormine) et la Nifédipine (Adalate) sur la fonction sexuelle de l'homme âgé : une étude contrôlée avec cross-over

D.L. MorissetTe, M.H. SkINNER, B.B. HoFFMAN, R.E. LEVINE ET J.M. DAVIDSON

Department of Molecular and Cellular Physiology, Stanford University, Stanford, California, USA

Arch. Sex. Beh., 22 : 99-108, 1993.

Les auteurs rapportent un essai contrôlé, de la fonction sexuelle de l'homme âgé hypertendu sous aténolol (Ténormine, Laboratoires ICI) et sous nifedipine (Adalate, Laboratoires Pfizer), dans une étude contrôlée contre placebo, randomisée avec cross-over.

L'étude a porté sur 16 hommes âgés de 60 à 75 ans (âge moyen 66.6 ans \pm 1.4 ) présentant une hypertension artérielle avec presssion diastolique entre 90 et $110 \mathrm{~mm}$ de mercure.

L'un des 2 traitements, aténolol ou nifedipine, leur a été attribué de façon randomisée avec des posologies de 30 à $90 \mathrm{mg}$ de nifedipine et de 25 à $100 \mathrm{mg}$ d'aténolone, jusqu'à normalisation des chiffres tensionnels.

L'évaluation de la fonction sexuelle des patients à porté sur 2 fois 2 semaines sous placebo, et 2 fois 4 semaines sous traitement.

La fonction sexuelle des patients a été évaluée par autoquestionnaires quotidiens portant sur 13 items : fréquence du désir, activité coïtale et non coïtale, fréquence et qualité des érections, fréquence et qualité de l'orgasme lors des rapports ou lors de la masturbation.

Hormis une diminution significative de la qualité des érections lors de la masturbation sous nifedipine, il n'y a eu aucune différence observée sous placebo et sous les 2 types de traitement, quelle qu'en soit la posologie.

Les auteurs concluent que les résulats de cette étude ne sont pas définitifs dans la mesure où le nombre de sujets étudiés était peu important. Cependant, il ne semble pas que les 2 drogues hypotensives utilisées aient altéré la fonction sexuelle des patients traités (M. BUVAT-HERBAUT). 


\section{CONTRACEPTION}

\section{Comparaison de deux androgènes asso- ciés à la medroxy-progestérone pour l'obtention de l'azoospermie chez des indonésiens}

World health organization task force on methods for the regulation of male fertility.

Fertil. Steril. Vol. 60 : 1062-1068, 1993.

La Contraception masculine hormonale qui s'était avérée insuffisamment efficace chez l'européen ou le nord américain, paraît pouvoir l'être chez certains asiatiques. C'est du moins la conclusion à laquelle arrive la Task Force de l'OMS, après une étude multicentrique sur 96 volontaires sains : $43 / 45$ hommes ayant reçu de la testostérone sous forme retard et $44 / 45$ de la 19 Nor T sous forme d'un ester, pendant 24 semaines, sont devenus azoospermiques. Les doses : $200 \mathrm{mg}$ toutes les semaines, pendant 7 semaines, puis $200 \mathrm{mg}$ toutes les 3 semaines, associés à $250 \mathrm{mg}$ de dépôt MPA toutes les 6 semaines.
Les 3 sujets "résistants" étaient cependant parvenus à des concentrations de 0,1 à 0,8 millions $/ \mathrm{ml}$. Les taux de FSH et LH se sont abaissés, mais il est impossible par les courbes de l'article de savoir si l'élévation qu'elles ont subie à la 24 ime semaine, avait lieu avant ou après la fin du traitement.

Sept hommes sous 19 nortestostérone et 2 sous testostérone se sont plaints d'une baisse du désir sexuel ce qui est à rapprocher des résultats obtenus chez les singes orchidectomisés et traités par 19 nortestostérone et chez lesquels il n'a pas été possible d'affirmer que l'on avait ramené de façon complète ou partielle la fonction sexuelle à la normale.

On a observé une réduction respectivement de $10 \%$ et $7 \%$ du volume testiculaire ( 19 nor T et $\mathrm{T}$ ). Les tests hépatiques sont restés stables. La principale limitation de la méthode tient dans le nombre élevé d'injections intra musculaires nécessaires.

Quant à la discordance entre les populations des différents continents étudiées, elle pourrait tenir à des facteurs nutritionnels, métaboliques ou génétiques, mais l'hypothèse n'a pas été soulevée par les auteurs du rôle des différences de gabarit des hommes : les plus grands indonésiens mesuraient $1 \mathrm{~m}$ 65 et pesaient 62,9 Kgs (moyenne 162 cms et $57,6 \mathrm{kgs})$ !(J. BELAISCH). 